\title{
Effect of the Growth Temperature in the Composition Fluctuation of GaInNAs/GaAs Quantum Wells
}

\author{
M. Herrera, ${ }^{* * *}$ Q. Ramasse ${ }^{* * *}$, D. González ${ }^{* *}$, R. García ${ }^{* *}$, N. Browning ${ }^{*, * * *}$, M.Hopkinson ${ }^{* * * *}$ and \\ H.Y.Liu
}

*Department of Chemical Engineering and Materials Science, University of California-Davis, One Shields Ave, Davis, CA 95616, U.S.A.

** Departamento de Ciencia de los Materiales e I.M. y Q.I., Universidad de Cádiz, Apartado 40, 11510 Puerto Real, Cádiz, Spain

${ }^{* * *}$ Lawrence Berkeley National Laboratory, National Centre for Electron Microscopy, 1 Cyclotron Road, Berkeley, CA 94720, U.S.A.

**** Lawrence Livermore National Laboratory, 7000 East Avenue, Livermore, CA 94550, U.S.A.

${ }^{* * * * *}$ Department of Electronic and Electrical Engineering, University of Sheffield, Mappin Street, Sheffield S1 3JD, United Kingdom

Dilute nitride GaInAs alloys have attracted the attention of many researchers for their application to long wavelength $(1.3-1.55 \mu \mathrm{m})$ laser diodes. The merit of this material is an unusually large negative band-gap bowing that allows the extension of the emission wavelength by adding small amounts of $\mathrm{N}$ to III-V alloys. The small size of $\mathrm{N}$ atoms also provides the possibility of reducing the lattice parameter of GaIn(N)As layers, thus reducing the lattice mismatch with GaAs substrates. However, the challenge in the growth of this alloy GaInNAs is that the solubility of $\mathrm{N}$ into GaInAs is very low [1], and consequently the material tends to phase separate.

The analysis by transmission electron microscopy of GaInNAs/GaAs(001) quantum wells grown at different temperatures in the range $375-420^{\circ} \mathrm{C}$ is reported here. Our results with the $220 \mathrm{BF}$ reflection have shown the existence of periodic strain contrast in all the wells (as can be observed in Fig. $1 \mathrm{a}$ and $1 \mathrm{~b}$ for the structure grown at $420^{\circ} \mathrm{C}$ ), associated with composition fluctuations in the alloy. These contrast variations are more pronounced on increasing the growth temperature, revealing a kinetic limitation in the phase separation. However, no noticeable variation of contrast has been found with the chemically sensitive 002DF reflection (Fig. 1c). The structure factor of the 002DF reflection under kinematical conditions depends mainly on the differences between the atomic scattering factors of III and V elements, being relatively independent of sample thickness for these contents [2]. The calculation of the structure factor of the GaInNAs alloy has shown that in order to obtain an invariant $002 \mathrm{DF}$ intensity profile it is necessary that any increase $\Delta \mathrm{y}$ in the $\mathrm{N}$ composition be accompanied by a simultaneous decrease in the In content $\Delta x \approx-2.21 \Delta y$. This suggests that in the analysed quantum wells there are two compositional modulation profiles, one for $\mathrm{N}$ and another for $\mathrm{In}, 90^{\circ}$ out of phase. The uncoupling of In and $\mathrm{N}$ composition profiles could explain the high strain fields that appear with 220BF reflection.

Initial work to analyse quantitatively the observed composition profiles using Z-contrast imaging and energy loss spectroscopy in a VG HB501 dedicated scanning transmission electron microscope are under way. It appears that contrast variations with a similar spatial frequency to that of the strain contrast micrographs can indeed be observed in the HAADF images (Fig. 2a and 2b). These initial results must however be taken with caution: strong contrast changes, possibly due to surface roughness, can be observed at higher magnification in both the wells and the barriers, thus making the definite interpretation of the oscillations present in Fig. $2 b$ rather difficult. Careful specimen preparation for future observations should therefore help confirming our preliminary conclusions. 


\section{References}

[1] I. H. Ho and G. B. Stringfellow, J. Cryst. Growth 178 (1-2) (1997) 1

[2] V. Grillo, M. Albrecht, T. Remmele, H. P. Strunk A. Yu. Egorov and H. Riechert, J. Appl. Phys. $90(2001) 3792$
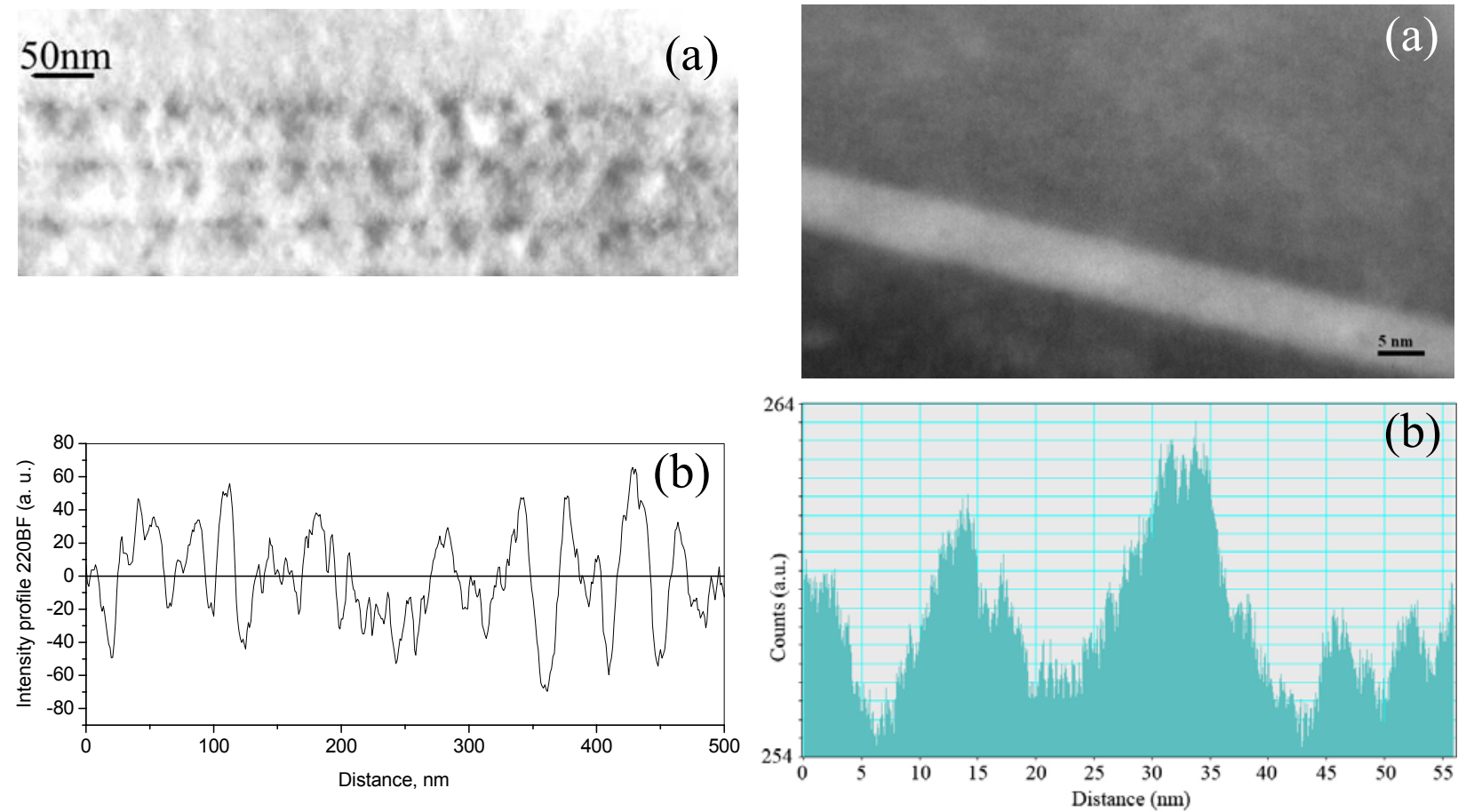

(c)

\section{$10 \mathrm{~nm}$}

Fig. 1: (a) 220BF micrograph of the sample grown

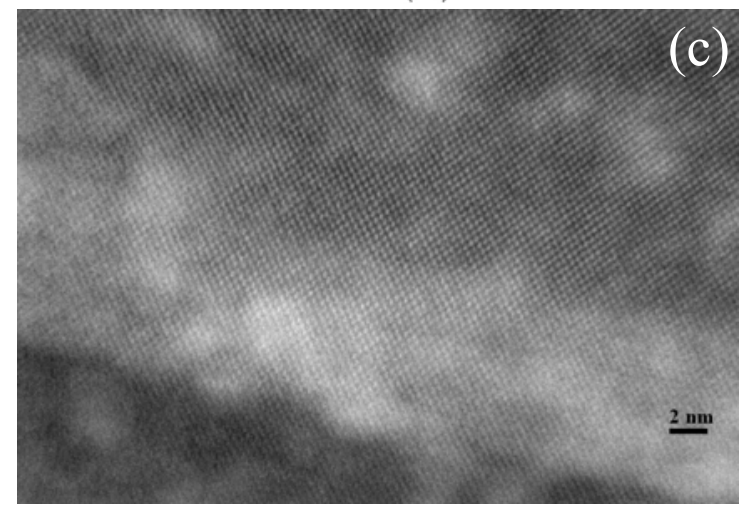

at $420{ }^{\circ} \mathrm{C}$; (b) intensity profile taken along the upper well in the micrograph shown in (a); (c) $002 \mathrm{DF}$ image of the sample grown at $420^{\circ} \mathrm{C}$.

Fig. 2:(a) HAADF overview of a well, with apparent contrast variations; (b) intensity profile taken along the well: the spatial frequency is similar to that observed in Fig. 1a; (c) high-resolution Z-contrast image, showing strong contrast changes in both well and barrier. 